\title{
Fault Tolerance \& Load Balanced Ad-hoc Networks using LRDV Routing Protocol
}

\author{
Sybi Cynthia J, R Rathna
}

\begin{abstract}
In mobile devices, location management is a major issue due to the change in location while connected to the network. In this paper we proposed a new routing protocol for sensor embedded mobile devices to localize the resection of exposure nodes over highly dynamic vector. It highly supports fault tolerance to avoid route breaks and it will reestablish new route before a route break. And also recover the fault based on direction and location. It Improves the quality of service $(Q o S)$ by minimizing the complexity of routing and it also highly supports fault analysis, speed of service, increased intelligence of tracking devices and speed recovery of fault over communication failures like breakage of links.
\end{abstract}

Index terms: location management, ad-hoc networks, routing protocols, QoS, fault tolerance.

\section{INTRODUCTION}

Wireless networks provide the users to exchange the data and acquisition at any time and any place. It has the function of connecting the moving states. It only works on presume based network establishment.

The nodes in the network must have cooperation among themselves to communicate throughout the network. Nonmilitary areas due to its automatic configuration and management capacities [1]. In ad-hoc network, there is no central system to manage the network. Ad-hoc networks do not require any preexisting network for communication so it is highly flexible[19]. If any terminal was beyond the range, the terminals act as a router so that the packets can reach from sender to the receiver. Mobile nodes coordination is must in order to have proper way of communication throughout the entire network [2].

Routing protocols want to perform four main functions such as maintaining connectivity of the network, transmission arrangement, channel assignment and packet routing. Routing protocols are classified either as routing strategy or network structure wise.

Revised Manuscript Received on July 13, 2019.

Sybi Cynthia J, Assistant Professor, Saveetha School of Engineering, Saveetha Institute of Medical and Technical Sciences, Chennai

R Rathna, Assistant Professor, Saveetha School of Engineering, Saveetha Institute of Medical and Technical Sciences, Chennai

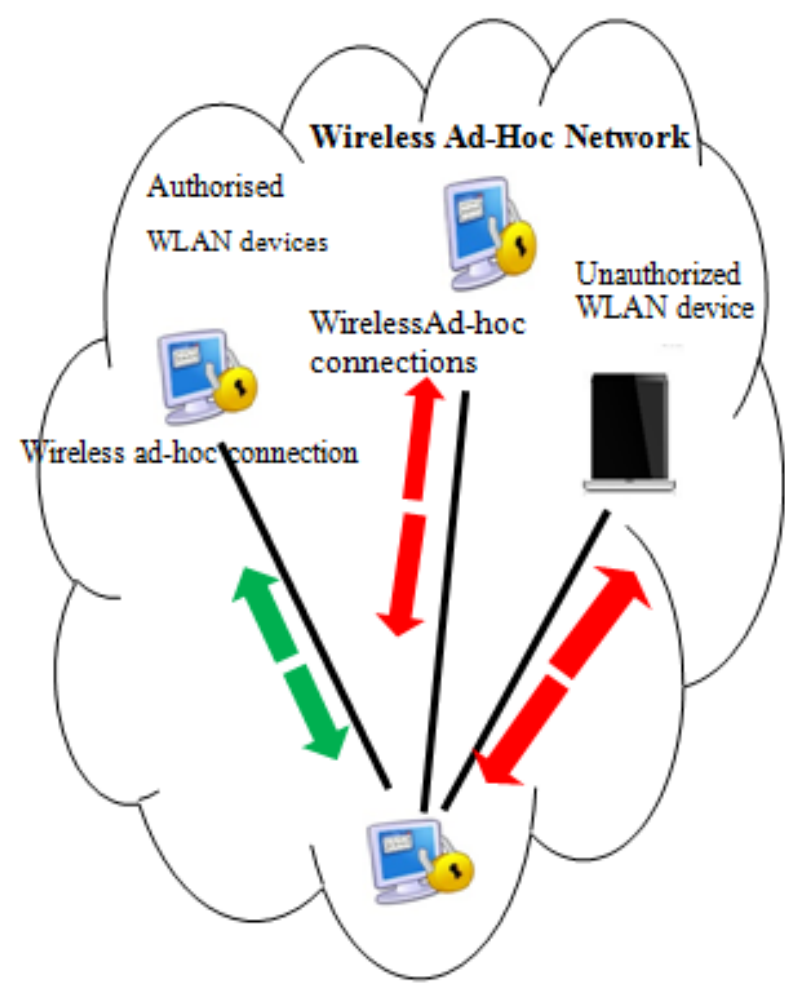

Fig. 1 Wireless ad-hoc network

The routing protocols are widely classified into three categories such as proactive routing protocols, reactive routing protocols, and location based routing protocols. Behavior of these protocols will get changed under different wireless conditions [7].

Well in proactive routing protocol, path information for each node (for source as well as destination) is maintained as well as the routing table entries will be updated periodically. The main advantage to the proactive routing protocol is availability of routes.

In reactive routing protocol its different, hence it only maintains the path information for destination node. But time consumption is more in this flooding technique because of redundant transmissions. Due to this, time taken for route establishment is more when compared to proactive routing protocol in MANET [6].

These challenges include Lack of visibility into the location of devices, Inefficient allocation and use of staff and equipment, Excessive cost of installing new equipment to offset losses and theft whereas security issues linked to the lack of insights, movement of devices, no central control mechanism and backbone network where wireless communication bandwidth is very limited. 
To handle the above issues in a simple way, smart mobility devices using an adaptive location magnitude grid protocol is used. Some of the applications of Location based system include military and government industries, emergency services and the commercial sector.

In this paper, we derived a location-tracking protocol, which is done by the cooperation of magnetometer sensors. Whenever a node is detected, it will be included into the virtual grid plane, on which the routing protocols are initialized to transfer the data. The communications which are coming out from the nodes will pass the virtual grid.

The rest of the paper is organized as follows. Section II reviews the work related to the proposed work. Section III briefs the problem statement of our work. Section IV describes the model of our network. Section V shows the location tracking of our work. Section VI examines the simulation outcomes and finally section VII concludes our proposed work.

\section{RELATED WORKS}

Some researches proposed a new routing protocol named as Reliable on-demand routing protocol (RORP) to compute the running span between two connected nodes in the network by using mobility prediction [12]. It also makes use of GPS (Global Positioning System) to estimate the time duration. Initially, it explores the request region in search of new routes in the network. Whenever the routing protocol finds a broken link, then the routing path is disconnected. This provides a back path for maintaining the route in case of broken routes.

Route strategy in this protocol is fully depend on weights to enhance the system and network's performance. Some factors are used to decide the route's weight. Traditional route discovery process discovers multiple alternative routes from source to destination. This protocol selects the largest weight path to reach the destination.

QoS metrics are mainly used for reserving the resources considering that MANET is does not have an infrastructure. It does not assume any predefined or specific MAC protocols[21].

Having multiple path to destination at intermediate nodes will help to reduce the route errors transmitted in route break recovery and also reduce overhead of additional route discovery. And also the performance of multipath routing protocol considerably increased than other existing routing protocols. Here in SMORT, the analysis is totally based on theoretical analysis of the network.

\section{PROBLEM STATEMENT}

One of the optimized versions of popular distributed bellman ford distance vector routing algorithm. Whenever there is a change in topology the nodes will broadcast routing messages and periodic updates will be exchanged with low frequency, but these results high overhead. For minimizing the overhead, routing protocols with link state exchange have been proposed. But for large networks, it is complicated since the movement of nodes trigger new updates.

\section{Objective}

In the proposed routing protocol, whenever the node is detected, it will be included in the virtual grid plane on which routing protocols are initiated to transmit the data. Virtual plane helps us to detect the nodes which moves in the same direction. Thus the proposed protocol does not have to consider the other neighboring nodes which are away from its direction. It reduces the bottlenecks in routing protocol and provides effective fault tolerance protocol.

\section{Virtual Plane}

Generally, magnetometers are used to monitor the directions. The inputs of all the magnetometer sensor nodes were placed in a 3D plane grid. In ad-hoc networks, each node must aware of its physical location as well as its neighbor's physical location in order to track the route. Each node has the ability to detect the existence of nearby moving nodes. In the proposed protocol, whenever the node is detects a new node or its neighboring node, then it will be included in the virtual plane grid. This virtual grid measures the direction with respect to the four basic directions (East, West, North and South) with 0 degree facing East and 180 degree facing the west. All the nodes in the grid maintain the location and selects the shortest path in the grid. Sensor nodes are arranged as an irregular network as shown in the figure 3 .

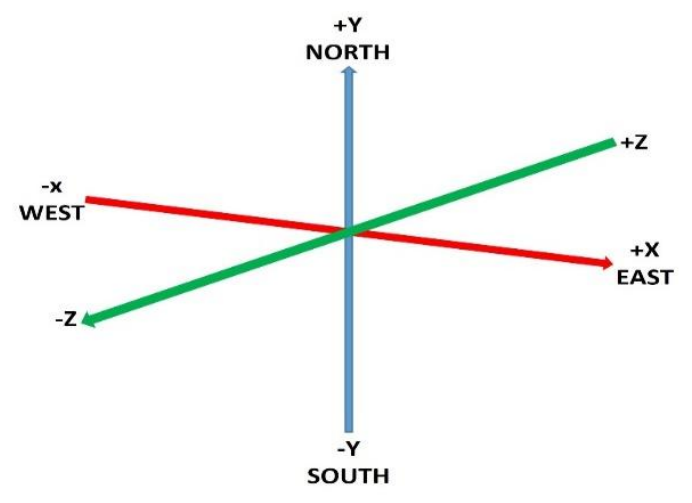

Fig. 2 Node's direction

\section{NETWORK MODEL}

This section shows the network model of our proposed work.

\section{Shortest Path}

Shortest path routing is the process of finding paths through a network which have a minimum of distance or other cost metrics. For example, routing of data packets on the internet which involves millions of routers in a complex, worldwide, multilevel network.

Mathematically, the shortest path is calculated by

$$
\operatorname{length}(p)=\sum_{i=1}^{k} w\left(v_{i-1}, v_{i}\right)
$$

Here $\operatorname{length}(p)=(v 0, v 1, \ldots v k)$ is sum of the weighted coordinates. 


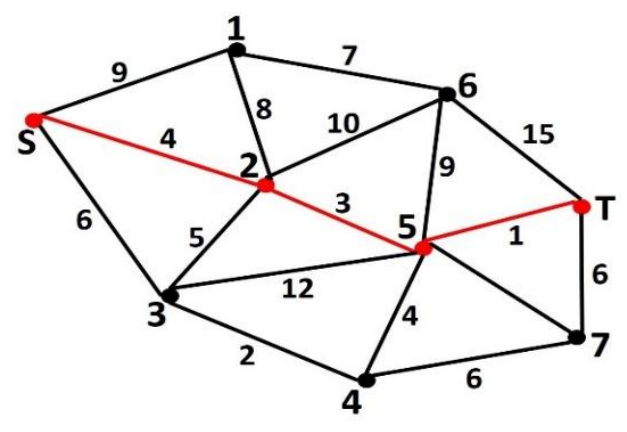

Fig. 3 Shortest path establishment

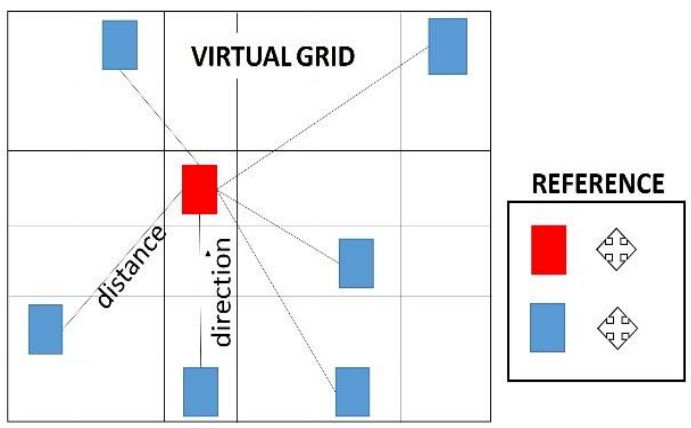

Fig. 4 Virtual Plane grid

\section{Routing}

The main aim of an ad-hoc networks routing algorithm is to deploy a route between a pair of nodes in the network precisely and efficiently. Because of its dynamic behavior, an ad-hoc network frequently changes location in its network topology. Consequently, the routing became challengeable and risky, and it has many known problems in ad-hoc networks.

Basically, the routing protocols use routing tables to store the information of nodes and to discover the node's location. The proposed routing protocol, LRDV uses the virtual grid to select the source path to transmit the net level node, which traverses in the same direction as source node. The virtual grid combines and reduces the service time with the help of routing table and location management.

\section{LOCATION TRACKING}

When two nodes begin to communicate, location tracking is used to track the nodes[14]. The nodes magnitude are retrieved from the virtual grid table and the point's direction is calculated by transmission of nodes from one to another. Based on the direction, the nodes are selected from the virtual grid.

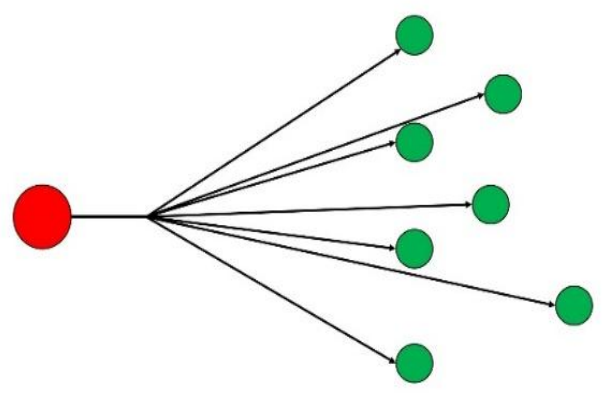

Fig. 5 Broadcasting multiple messages
In this way the random selction of nodes are stopped and proper nodes are selected, which are in the right direction[15]. The communiction speed will be increased and the usuage of the ad hoc networks get minimized. This method will support network's overhead due to the broadcast of unwanted node search in the network[8][9] as shown in the figure.

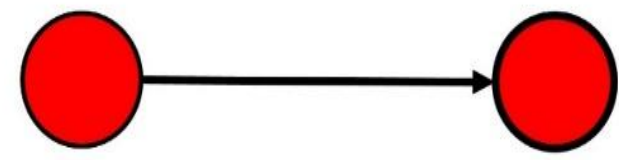

Fig. 6 Broadcasting single message

\section{SIMULATION RESULTS}

This section examines the outcomes of the proposed work. The evaluation is done based on metrics, such as closeness of the simulation outcomes to the theoretical predictions.

\section{Simulation Framework}

We used the network simulator as the basic platform on which our simulations were explained. We have used $600 \times 600$ grid with 20 sensor nodes as shown in the figure.

We assigned unique id to each node, and the nodes reading was a random integer uniformly distributed in the grid with the specific range. In each turn, the nodes are selected using the shortest path routing protocol.

The simulation of LRDV protocol applied on ad-hoc network terminal is used to execute the code. This window displays the channel and other relevant details of the code. Once the code is successfully generated then the simulation runs in another window. Figure 9 shows the simulation results of LRDV protocol applied on 40 nodes in the network.

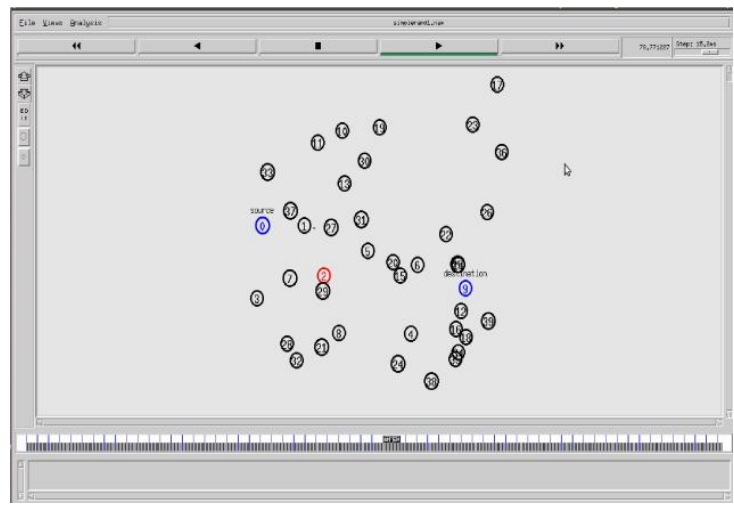

Fig. 7 Simulation Result of 40 Nodes

There are forty nodes in this region and the node zero is labelled as source with differentcolour. The colour blue is used to differentiate it from other nodes. And the node 9is labelled as destination node. This node is also given blue to show that it is different fromothers. The nodes are not connected by any links since it is wireless. 
The nodes are scattered everywhere since it has no infrastructure to establish the connection. Whenever the node embedded with magnetometer discovered the next node within its range it places the nodein the $3 \mathrm{D}$ virtual plane next to the source node.

LRDV protocol unicasts the message to the other nodes. The simulations use 20 nodes and it uses the channel type is "wireless channel".

The MAC type is "MAC/802_11" and the interface queue type is "CMUPriQueue".The Radio propogation model is "Propogation /Two-way Ground.

Table I shows the comparison of AODV and LRDV by pausing the simulation time. The time is analysed to estimate the performance of both the routing protocols.

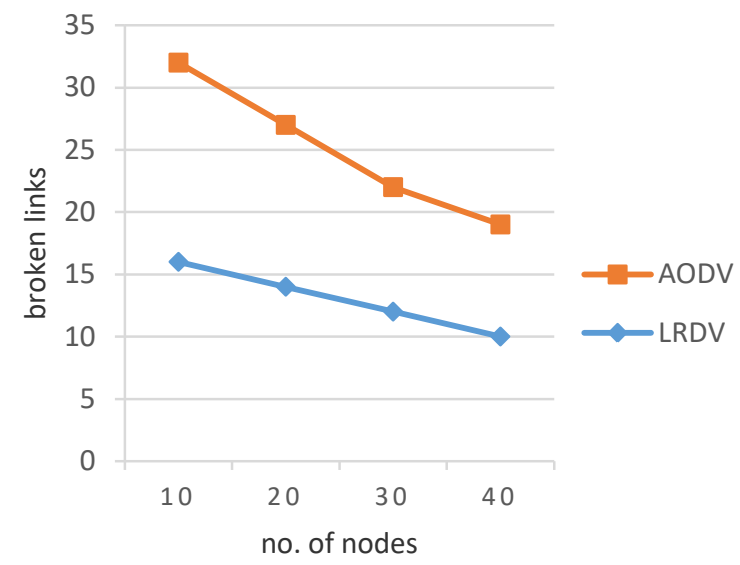

Fig. 8 Performances Analysis

Figure 10 shows the performance graph of LRDV compared to AODV. X-axis contains the value of number of nodes and $\mathrm{Y}$-axis contains the value of broken links in the network. The performance figure concludes that the proposed method LRDV outperforms AODV. LRDV considerably reduces the broken links than AODV.

Table. 1 Comparison of AODV and LRDV

\begin{tabular}{|l|l|l|l|l|l|l|}
\hline $\begin{array}{l}\text { Pauseti } \\
\text { me }\end{array}$ & \multicolumn{2}{|l}{ AODV } & \multicolumn{2}{l|}{ LRDV } \\
& \multicolumn{2}{|l|}{} \\
\hline & 1 & 2 & 3 & 1 & 2 & 3 \\
\hline 20 & 377.9 & 67.48 & 98.8 & 360.8 & 57.38 & 96.8 \\
& 4 & & 73 & 4 & & \\
\hline 40 & 349.9 & 107.2 & 98.0 & 320.8 & 99.14 & 94.5 \\
& 2 & 8 & 3 & 8 & & \\
\hline 60 & 324.5 & 124.2 & 104. & 302.5 & 115.2 & 102.6 \\
& 4 & 65 & 92 & 0 & 6 & 4 \\
\hline 80 & 376.6 & 147.9 & 107. & 355.5 & 127.9 & 103.2 \\
& 7 & 12 & 22 & 7 & 1 & 5 \\
\hline
\end{tabular}

The term fault tolerance is evaluated in terms of the faults tolerated in number of nodesagainst time in milliseconds. When a fault node is identified in mobile Ad hoc network, theprotocols AODV, DSR sends back RERR message to source node. The source node informsall other nodes about the fault. And the route request process continues again in order tosearch for a new route. Hence it takes lot of time to tolerate the fault.

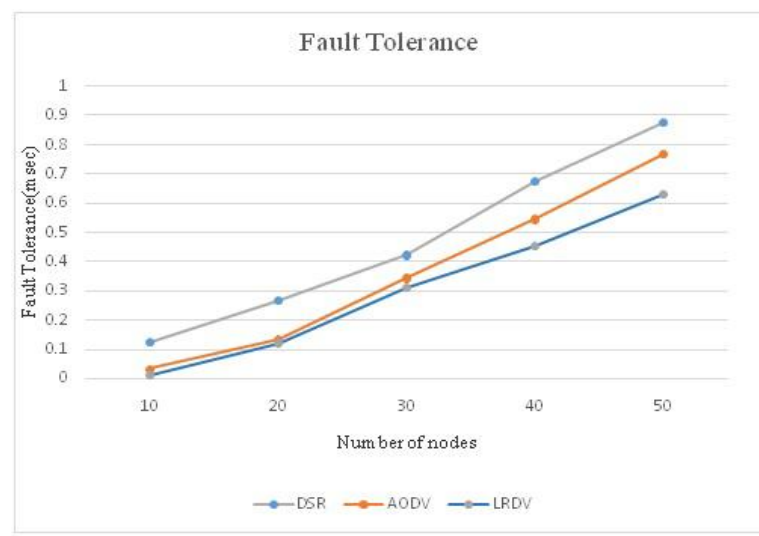

Fig. 9 Analysis of Fault Tolerance in LRDV

In LRDV the shortest nodes are placed in the virtual plane grid. When a node isidentified as fault node, the protocol directly chooses the next node which is in the grid.

Hence LRDV tolerate the fault in less time. The Figure 11 shows the analysis of Fault Tolerance in LRDV. Here the number ofnodes are compared against time in milliseconds. It shows that when the numbers of nodesare increasing the fault is also increasing. And it is tolerated by LRDV routing protocol inless time when it is compared with other routing protocols AODV and DSR.

\section{CONCLUSION}

In this paper, we explained the study of location management in wireless ad hoc network to improve the routing by achieving good performance in terms of QoS. In addition to that, our algorithm is local and deals with dynamicbehavior of the networks efficiently. The outcomes of this paper mainly focuses on location management among the mobile ad hoc networks. It also gives the successive outcomes of routing and broadcast. Most of the methods come with various sensors deployed on the locations, position and identifies the limitiations on those areas. Generally, it is not useful for general purpose among the mobile ad hoc devices. The proposed work is a successive model among the emerging mobile ad hoc networks and it deals with magnetometer to track the location efficiently.

The advantages of this proposed system will improve the quality of services, minimize the complexity of routing, highly support for fault analysis, clustering, speed of service, increased intelligence of tracking devices, speed recovery of fault over communication failures like link breakage and fast relocation. Also we used the shortest path routing based on virtual grid. The virtual gird consists of all nodes and the shortest path routing is applied on it. This routing is used to select the shortest path available in the network. Our study shows that the outcomes of the shortest path routing depend upon the selected parameters and the selected protocol used for routing. 


\section{REFERENCES}

1. Christine T. Cheng, H. L. Lemberg, Sumesh J. Philip, van den Berg .E, and Zhang T, "SLALoM: a scalable location management scheme for largemobile ad-hoc networks," Proceedings of Wireless Communications and Networking Conference", March 2002.

2. David Remondo, Ignas G. Niemegeers, "Ad hoc networking in future wireless communications", 26 February 2002.

3. Kaouthar Sethom, Khaled Masmoudi, Hossam Afifi,"A Secure P2P Architecture for Location Managemen”., ACM, May 2005, pp. 22-26.

4. Kevin Lee, Hong Wing Lee, Sanjay Jha, NirupamaBulusu, "Adaptive Distributed Location Management in Mobile, Wireless Networks", IEEE Communications Society, 2004.pp. 4077-4081.

5. Lenders V, May M, Member, IEEE, and B.Plattner, Member, IEEE, "Density-Based Anycast: A Robust Routing Strategy for Wireless Ad Hoc Networks", IEEE/ACMTRANSACTIONS ON NETWORKING, VOL. 16, NO. 4, AUGUST 2008.

6. Mohapatra .S, Kanungo .P, "Performance analysis of AODV, DSR, OLSR and DSDV Routing Protocols using NS2 Simulator", 2005,pp.123-135.

7. MacLean S, Datta S,'Reducing the Positional Error of ConnectivityBased Positioning Algorithms Through Cooperation Between Neighbors, Mobile Computing”, IEEE Transactions on (Volume:13, Issue: 8 ), Page(s):1868 - 1882, DOI:10.1109/TMC.2013.143.

8. Mario J.N, Lu I.T, "A peer-to-peer zone-based two-level link state routing for mobile adhoc networks", IEEE Journal on Selected Areas in Communications 17 (8) (1999) 1415-1425.

9. Nen-Chung Wang, Shou-Wen Chang," A reliable on-demand routing protocol for mobile ad hoc networks with mobility prediction", 2 June 2005 .

10. Naveed Ahmad, Naveed Riaz, Mureed Hussain,"Ad hoc wireless Sensor Network Architecture for Disaster Survivor Detection", International Journal of Advanced Science and Technology, Vol. 34, September, 2011.

11. Nen-Chung Wang, Yung-Fa Huang, Jhu Chan Chen, "A stable weightbased on demand routing protocol for mobile adhoc networks", Elsevier, Information sciences 177(2007)pp.5522-5537

12. Remondo Bueno .D, K. Coreman, R. Tafazolli, "Adhoc networks, in:Proceedings of the Second WWRF Meeting", Helsinki, Finland, May10-11, 2001

13. Royer E.M, Toh C., "A review of current routing protocols for adhoc mobile wireless networks", IEEE personal communications,1999, pp 46-56.

14. Reddeppa Reddy L, S.V. Raghavan, "SMORT: Scalable multipath on demand routing for mobile adhoc networks", Elsevier, Ad Hoc networks 5(2007)pp.162-188.

15. Sayed .E, "Shortest Path Routing paths in MANETs", Natural Science Publishing Cor., September 2016.

16. SubhansuBandyopadhyay, KoushikMajumder, BaishaliGhosal, "A CircularLayer Location Management Scheme for Mobile Adhoc Networks", 2006

17. Valera A.C, Student Member, IEEE, Winston K.G. Seah, Senior Member, IEEE, and S.V.Rao, Senior Member, IEEE, "Improving Protocol Robustness in Ad Hoc Networks throughCooperative Packet Caching and Shortest Multipath Routing", IEEE TRANSACTIONS ONMOBILE COMPUTING, VOL. 4, NO 5, SEPTEMBER/OCTOBER 2005.

18. Ying Dong, Tat Wing Chim, Victor O.K. Li, S.M. Yiu, C.K. Hui, "ARMR: Anonymous routing protocol with multiple routes for co", 23 April 2009.

19. Shuib Basri, D. Yuvaraj, "Prevention, Reduction and Recognition of Wormhole Attack by Coordination in the Wireless Adhoc Network", International Innovative Research Journal of Engineering and Technology, 4(3), 2019

\section{AUTHORS BIOGRAPHY}

J. Sybi Cynthia. completed her Master of Engineering in

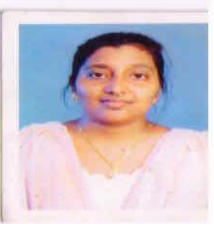
Computer Science and Engineering, in 2012 from The Rajaas Engineering College, Tirunelveli, India affiliated to Anna University, Chennai. She received the $\mathrm{PhD}$ in Computer Science and Engineering discipline. At present working as Asst. Professor in the Department of Computer Science and Engineering in Saveetha School of Engineering, Saveetha Institute of Medical and Technical Sciences. Her main interest and work areas are Network Security and Wireless Sensor Network.

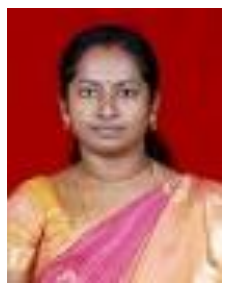

Dr.R.Rathna has finished her B.E in Information Technology from Bharathidasan University in the year 2004, M.Tech in Information Technology in the year 2007 from Sathyabama Institute of Science and Technology and Ph.D in Wireless Sensor Networks in the Faculty of Computer Science and Engineering in the year 2015 from Sathyabama Institute of Science and Technology. She is having 12 years of experience in Teaching. She worked as Assistant Professor in the Faculty of Computer Science and Engineering in Sathyabama Institute of Science and Technology, Chennai and as Associate Professor in the Department of Computer Science and Engineering in Saveetha School of Engineering, Chennai. 